globin, cytochromes or catalase was chosen for its light-absorption properties. Similarly the 'primitive' pigment of an ancestral photosynthesizer need not initially have been selected for optimum absorption of light. Clearly the bioenergetic function of even 'modern' chlorophyll depends as much on its properties as a lipid-soluble electron carrier as on its absorption of light energy ${ }^{2}$.

Once a line of organisms opts for a metalloporphyrin structure to carry out any function, however, it is limited by the chemical properties that arise from the high symmetry of the underlying conjugated carbon skeleton. In chemical terms symmetry is expressed as degeneracy in the energy states available to a molecule. Such degeneracies are the starting point for the now classical analysis of metalloporphyrin spectra ${ }^{3}$. This describes how the two-band spectrum of a metalloporphyrin arises: a strongly allowed ultraviolet-blue absorption (labelled B) and a weak green absorption that is formally forbidden (labelled Q). The breakdown of the strict symmetry of the molecule is expressed by Gouterman's parameter $v$. The square of the dipole moment $\left(r^{\prime}\right)$ for the weak green absorption determines the intensity of the absorption according to

$$
\left(r^{\prime}\right)^{2}=(v R-r)^{2}
$$

where $R$ and $r$ are the dipole moments associated with the $B$ and $Q$ transitions, respectively, in the perfectly symmetrical molecule and $v$ is a mixing parameter. From this it is clear that a breakdown in symmetry mixes the intensity of the B transition into the weak $Q$ to an extent controlled by $v$. But this is exacted at a price. The separation in energy between the bands is

$$
2 A_{1 \mathrm{~g}}^{\prime \prime}(1+v)^{2}
$$

where the factor $A_{10}^{\prime \prime}$ is a parameter expressing the fairly constant shift in the energies of excited states by interelectronic repulsion.

In this quantum-mechanical system, then, contrary to 'common sense' the borrowing of intensity from the strong $B$ by the weak visible $Q$ absorption increases as the separation between them increases. The red-shift of a metalloporphyrin visible band is connected with its increased intensity $v$. To achieve a reasonable visible intensity chlorophyll breaks the metalloporphyrin symmetry by substituting a saturated $\mathrm{C}-\mathrm{C}$ bond where the conjugation strictly requires partial double-bond character. According to the expressions above this cannot help but shift the $\mathrm{Q}$ band from the green into the red - giving a pigment that is green to the eye.

Too great a shift into the red, however, can yield a pigment lacking an excited state that is energetic enough for the photolysis of water; a blind alley now occupied by prokaryotes using bacteriochlorophyll, which contains two conju- gation-breaking saturated $\mathrm{C}-\mathrm{C}$ bonds ${ }^{4}$.

It seems plausible, then, that chlorophyll represents a compromise where a metalloporphyrin's visible absorption is maximized without losing the ability for oxygenic photosynthesis so that the unique properties of the metalloporphyrin electronic system as a biological electron 'solvent' can be effectively exploited.

School of Biological Sciences,

University of East Anglia,

Norwich NR4 7TJ, UK

1. Goldsworthy. A. Nature 328, 207-208 (1987)

. Nicholls, D.G. Bioenergetics (Academic, London, 1982)

3. Gouterman, M. J. chem. Phys. 30, 1139-1161 (1959)

4. Olson, J.M. Science 168, 438-446 (1978).

\section{LHRH agonists and human breast cancer cells}

SIR-The use of luteinizing hormone releasing hormone (LHRH) agonists to achieve medical castration in patients with metastatic breast and prostate cancer has been widespread in recent years. The primary mechanism by which these compounds block steroid hormone production by the gonads is by inhibition of the release of luteinizing hormone by the pituitary. Miller et al. ${ }^{1}$ raised the issue of direct inhibitory effects of the LHRH peptides on breast cancer cells. Low-affinity binding activity in malignant but not normal breast tissue has also been reported ${ }^{2.3}$. In studying the effects of a variety of LHRH-related peptides on the growth of MCF-7 hormone-responsive breast cancer cells in vitro, however, we have been unable to reproduce the original inhibitory effects ${ }^{1}$.

Experiments designed to duplicate the treatment conditions used by Miller ${ }^{1}$ did not reveal any inhibitory effects of LHRH or related peptides on MCF-7 cell growth No change in the doubling time of the MCF-7 cells was caused by any of the compounds studied (see table). There was no effect by Leuprolide (leuprovelin) even when used at a concentration as high as $10^{-6} \mathrm{M}$. Because of the lack of inhibitory effects of any compound tested, many other treatment schedules and media conditions were tested. They included treatment every day, every other day and every third day with or without concurrent media changes and treatment in media supplemented with $5 \%$ charcoal-stripped, sulphatase-treated calf serum with or without oestradiol $\left(10^{-8} \mathrm{M}\right)$ added to the media. No inhibition was observed.

These observations suggest that clonal variation among $\mathrm{MCF}-7$ cells alters their response to LHRH and its related peptides. In addition, because of the high concentrations of peptides required for inhibition, when it is observed, and the low affinity $\left(K_{\mathrm{d}}=10^{-6} \mathrm{M}\right)$ of LHRH binding $^{2.3}$, it is unlikely that LHRH agonists exert significant direct biological effects on breast cancer cells in vivo. Perhaps the

\begin{tabular}{|cc}
\hline \multicolumn{2}{c}{ Doubling times of cells } \\
\hline \multicolumn{1}{c}{ Treatment } & $\begin{array}{c}\text { Doubling time } \\
\text { (\% control) }\end{array}$ \\
Control & 100 \\
LHRH $\left(10^{-7} \mathrm{M}\right)$ (Sigma) & 93 \\
Leuprolide $\left(10^{-6} \mathrm{M}\right)$ (Tap) & 93 \\
$\left(10^{-7} \mathrm{M}\right)$ & 100 \\
$\left(10^{-9} \mathrm{M}\right)$ & 100 \\
$\left(10^{-12} \mathrm{M}\right)$ & 110 \\
Buserelin $\left(10^{-7} \mathrm{M}\right)($ Hoechst $)$ & 100 \\
L2636 $\left(10^{-7} \mathrm{M}\right)$ (Sigma) & 100 \\
L4261 $\left(10^{-7} \mathrm{M}\right)$ (Sigma) & 107 \\
\hline
\end{tabular}

Cells were grown in Dulbecco's minimal essential medium supplemented with $10 \%$ fetal bovine serum (FBS), gentamicin $\left(40 \mathrm{mg} \mathrm{t}^{-1}\right)$ and glutamine $\left(0.6 \mathrm{~g} \mathrm{l}^{-1}\right)$. Medium was changed daily and treatment substance added daily. Cells were counted every other day by cell counter (Particle Data). No insulin was added to the media. Control cell doubling times ranged from 24 to $33 \mathrm{~h}$ in the presence of FBS and phenol red. Time points for each treatment were performed in triplicate.

observations of Miller et al.' and Eidne and co-workers ${ }^{2.3}$ are best viewed as insights into the possibility of an autocrine regulatory function for peptide hormones in mammary cells.

\section{G. WILDING M. CHEN \\ E.P. GelmanN}

Medicine Branch,

\section{National Cancer Institute,}

Bethesda, Maryland 20892, USA

1. Miller, W.R., Scott, W.N., Morris, R., Fraser, H.M. \& Sharpe, R.M. Nature 313, 231 (1985)

2. Eidne, K.A., Flanagan, C.A. \& Millar, R.P. Science 229 989 (1985).

3. Eidne, K.A., Flanagan, C.A., Harris, N.S. \& Millar, R.P J. clin. Endocr. Metab. 64, 425(1987).

Miller RePlies - Several groups have contacted me indicating an inability to reproduce our results in their laboratories and I think that, as Wilding et al. suggest, clonal variation of cells and culture conditions can in part explain these discrepancies. Certainly, we have in our possession other MCF-7 cells which do not respond in the same way to LHRH agonist and we have reported that, in the presence of insulin, it is difficult to show effects of LHRH agonists (W.R. Miller et al., Monogr. Ser. EORTC, Vol. 18). An exchange of cells with Wilding is in progress to resolve these issues.

Nevertheless, there are other reports of direct effects of LHRH analogues on breast cancer cells. Thus Blankenstein and co-workers (Eur. J. Cancer clin. Oncol. 21, 1493; 1985) showed inhibitory effects on MCF-7 cells; Wiznitzer and Benz (Proc. Ann. Am. Ass. Cancer Res. $25,208 ; 1984)$ inhibitory effects on T-47D cells and, as Wilding et al. point out, Eidne et al. reported inhibitory effects of LHRH antagonists on several cell lines.

$$
\text { W.R. MILLER }
$$

University Department of Clinical Surgery, Royal Infirmary, Edinburgh

EH3 9YW, UK 